\title{
ON WEIGHTED SPACES WITHOUT A FUNDAMENTAL SEQUENCE OF BOUNDED SETS
}

\author{
J. O. OLALERU \\ Received 5 February 2001 and in revised form 21 September 2001
}

\begin{abstract}
The problem of countably quasi-barrelledness of weighted spaces of continuous functions, of which there are no results in the general setting of weighted spaces, is tackled in this paper. This leads to the study of quasi-barrelledness of weighted spaces in which, unlike that of Ernst and Schnettler (1986), though with a similar approach, we drop the assumption that the weighted space has a fundamental sequence of bounded sets. The study of countably quasi-barrelledness of weighted spaces naturally leads to definite results on the weighted (DF)-spaces for those weighted spaces with a fundamental sequence of bounded sets.
\end{abstract}

2000 Mathematics Subject Classification: 46A08, 46E30.

1. Introduction and notations. The countably barrelledness, countably quasi-barrelledness, barrelledness, and quasi-barrelleness of the space $C(X)$ of continuous functions on a completely regular Hausdorff space $X$ equipped with the compact open topology (c-op), including when it is a (DF)-space and ( $g \mathrm{DF})$-space, is well known (cf. $[7,13])$. In the more general setting of weighted spaces, Ernst and Schnettler [3] studied the $(g \mathrm{DF})$ and the quasi-barrelledness of weighted spaces by constructing a Nachbin family on $X$ which is based on the assumption that the weighted space has a fundamental sequence of bounded sets. A weighted space need not have a fundamental system of bounded sets. There are classical examples of such spaces, for example, let $X$ be a noncompact locally compact and $\sigma$-compact space, and let $C(X)$ be the space of all continuous real-valued functions on $X$ equipped with the compact-open topology. $C(X)$ is a weighted space [14], metrizable and not normable [11, Observation 10.1.25] and hence it does not have a fundamental sequence of bounded sets [6]. See also [10, page 9] for another example. Following the same approach, the countably barrelledness and barrelledness of weighted spaces was studied in [10] without assuming that the weighted space has a fundamental sequence of bounded sets. This paper is a follow up of [10] although we have made it independent of it. We characterise countably quasi-barrelled (Section 3) and quasi-barrelled (Section 4) weighted spaces by a constructed Nachbin family on $X$ without assuming that the spaces have a fundamental sequence of bounded sets. This approach makes the study of (DF)-weighted spaces easy as it can be seen in Section 5. In Section 6, we show that every countably quasi-barrelled weighted space satisfies the countable neighborhood property (cnp).

As an application, we recover the known results for the countably quasi-barrelledness and quasi-barrelledness of $C(X)$ equipped with compact open topology (Corollary 3.10) and at the same time get new results for the space of bounded continuous 
functions on $X$ equipped with the strict topology (cf. Corollaries 3.13 and 4.3). Also [1, Theorem 1.8] on the (DF)-weighted inductive limits was recovered as a corollary to Proposition 4.5.

We adopt the notation and definitions of weighted spaces (cf. [1, 3, 12]).

Let $X$ denote, unless otherwise indicated, a completely regular Hausdorff space and $E$, a normed linear space. $F(X, E)$ is the vector space of all mappings from $X$ to $E$, $C(X, E)$ is the vector subspace of all continuous mappings from $X$ to $E$. $B(X, E)$ is the vector subspace of all mappings from $X$ into $E$ such that $f(X)$ is a bounded subset of $E$, and $B_{0}(X, E)$ is the vector subspace of $B(X, E)$ consisting of those bounded mappings $f$ from $X$ into $E$ that vanish at infinity, that is, those $f \in B(X, E)$ such that, for any $\epsilon>0$, there is a compact subset $K \subset X$ such that $\|f(x)\| \leq \epsilon$ for every $x \in X$ outside of $K$. The vector subspace $C(X, E) \cap B(X, E)$ is denoted by $C_{b}(X, E)$, and $C(X, E) \cap B_{0}(X, E)$ is denoted by $C_{0}(X, E)$. The set $C_{C}(X, E)$ denotes the subset of $C(X, E)$ consisting of those functions that are identically zero in $E$ outside of some compact subset of $X$. If each function is complex valued, we write the corresponding spaces omitting $E$.

A nonnegative real-valued upper semicontinuous function (usc) on $X$ is called a weight. If $U$ and $V$ are two sets of weights on $X$, we write $U \leq V$ whenever, given $u \in U$, there is $u, v \in V$ such that $u \leq v$ (pointwise on $X$ ). We write $U \sim V$ if and only if $U \leq V$ and $V \leq U$. A set of weights $V$ on $X$ is said to be directed upwards if for $v_{1}$, $v_{2}$ in $V$ and every $\lambda>0$, there is $v \in V$ such that $\lambda v_{i} \leq v, i=1,2$. If a set of weights $V$ on $X$ is directed upwards and $V>0$, that is, if, given any $x \in X$, there is $v \in V$ with $v(x)>0$, then $V$ will be referred to as a Nachbin family on $X$.

Let $V$ be a Nachbin family on $X$ such that, for every $x \in X$, there is an $f \in F V(X, E)$ such that $\|f(x)\| \neq 0$, then we define the following weighted spaces:

$$
\begin{aligned}
F V(X, E) & =\{f \in F(X, E):(v f)(X) \text { is bounded in } E \forall v \in V\}, \\
F V_{0}(X, E) & =\{f \in F(X, E): v f \text { vanishes at infinity } \forall v \in V\} .
\end{aligned}
$$

We consider $F V(X, E)$ endowed with the locally convex topology $w_{v}$ defined by the family of seminorms

$$
p_{v}(f)=\sup _{x \in X} v(x)\|f(x)\|, \quad v \in V
$$

with a basis of neighborhoods of the origin of the form

$$
V_{v}=\left\{f \in F V(X, E): p_{v}(f) \leq 1, v \in V\right\}
$$

Clearly $F V_{0}(X, E)$ is a closed subspace of $F V(X, E)$.

Examples of those spaces are found in [13]. Furthermore, we will denote $C V(X, E)$ and $C V_{0}(X, E)$, respectively, as $F V(X, E) \cap C(X, E)$ and $F V_{0}(X, E) \cap C(X, E)$ each equipped with the topology induced by $w_{v}$.

We recall that a subset $M \subset F V(X, E)$ is full if

$$
M=\{f \in F V(X, E):\|f\| \leq \mu\}, \quad \mu(x)=\sup _{f \in M}\|f(x)\| \forall x \in X
$$


In the sequel, we put $a+\infty=\infty$ for $n \in \mathbb{R}^{+} \cup \infty, 1 / \infty=0,1 / 0=\infty$.

2. Preliminary lemmas. The following definitions are recalled for reference. For the definitions of other terminologies mentioned below, see [4].

Definition 2.1. A subset of a locally convex space is called a barrel if it is absolutely convex, absorbent, and closed.

DEFINITION 2.2. A locally convex space $E$ is called bornivorous if $B$ absorbs all bounded subsets of $E$.

DEFINITION 2.3. A locally convex space is called barrelled (quasi-barrelled) if every barrel (bornivorous barrel) is a neighborhood of zero.

The following lemma, which was proved by Bonet [1, Lemma 1.2] for $C V(X, E)$, is also true for $F V(X, E)$.

LEMmA 2.4. Let $V$ be a Nachbin family on $X$ for the weighted space $F V(X, E)$ such that $v, w \in V$, then, $V_{v} \leq V_{w}$ if and only if $w(x) \leq v(x)$ for every $x \in X$.

Following the argument of the proof of [14, Theorem 3.1], if $X$ is a locally compact space or $V \leq B_{0}(X)$, then Lemma 2.4 holds for $F V_{0}(X, E)$ and thus we have the following result.

LEMMA 2.5. Let $V$ be a Nachbin family on $X$ for the weighted space $F V_{0}(X, E)$ such that $V \leq B_{0}(X)$ or $X$ is locally compact. If $v, w \in V$, then $V_{v} \leq V_{w}$ if and only if $w(x) \leq$ $v(x)$ for every $x \in X$.

The following lemma, which was proved for $C V(X)$ in [3, Lemma 1.3], is also true for $F V(X, E)$, and of course true for any of the weighted spaces $F V_{0}(X, E)$ and $C V_{0}(X, E)$.

LEMMA 2.6. Let $\left\{M_{i}, i \in \mathbb{N}\right\}$ be a sequence of full subsets of the space $F V(X, E)$ and $\mu_{i}(x)=\sup _{f \in M_{i}}\|f(x)\|$. Then $M=\bigcap M_{i}$ is a full subset of $F V(X, E)$ and $M=\{f \in$ $\left.F V(X, E):\|f(x)\| \leq \inf _{i} \mu_{i}(x)\right\}$.

The following lemmas are very important for our work.

LEMMA 2.7. Let $V$ be a Nachbin family on $X$ and $s$ an arbitrary function from $X$ to $[0, \infty]$ such that $1 / s \in B_{0}(X)$ and the set $M=\{f \in F V(X, E):\|f\| \leq s\}$ is absorbent in $F V(X, E)$. Then $M$ is bornivorous. Suppose that $\inf _{f \in M}\|f(x)\|$ is usc on $X$, then there is a smallest weight $v_{s}>1 / s$ and $M=V_{v_{s}}$.

Proof. In view of [3, Lemma 1.5], it is sufficient to show that $M$ is bornivorous. This is immediate since $f \cdot 1 / s$ is bounded on $X$.

Similarly, in view of Lemma 2.5, we have the following lemma.

LEMmA 2.8. Let $V$ be a Nachbin family on $X$ such that $X$ is locally compact or $V \leq$ $B_{0}(X)$. If $s$ is an arbitrary function from $X$ to $[0, \infty]$ such that $1 / s \in B_{0}(X)$ and the set $M=\left\{f \in F V_{0}(X, E):\|f\| \leq s\right\}$ is absorbent in $F V_{0}(X, E)$, then $M$ is bornivorous. Suppose that $\inf _{f \in M}\|f(x)\|$ is usc on $X$, then there is a smallest weight $v_{s}>1 / s$ and $M=V_{v_{s}}$. 


\section{Countably quasi-barrelledness of weighted spaces}

DEFINITION 3.1. Let $V$ be a Nachbin family on $X$, we define a new Nachbin family $V^{*}$ on $X$ by adjoining the smallest weights (including all their multiples) greater than the suprema of all countable weights in $V$ (with the condition that the suprema of all such weights vanish at infinity) to $V$.

LEMMA 3.2. Let $V$ be a Nachbin family on $X$ and let $\left(v_{n}\right)_{n}$ be the set of all sequences $\left(v_{n}\right)$ in $V$ such that $\sup _{n} v_{n} \in B_{0}(X)$ for each $\left(v_{n}\right) \in V$. Let $v_{s(n)}$ be the smallest weight greater than $\sup _{n} v_{n}$ for each $\left(v_{n}\right) \in V$. Then $V^{*}$ is the system of all positive multiples of $v_{s(n)}$ as $\left(v_{n}\right)$ runs through all sequences in $V$.

REMARK 3.3. Suppose that $U$ is a bornivorous barrel in $F V(X, E)$ such that $U=$ $\cap\left\{U_{n}, n \in \mathbb{N}\right\}$, where each $U_{n}$ is a closed and absolutely convex neighborhood of zero in $F V(X, E)$. Then for each $U_{n}$, there is a $v_{n}$ in $V$ such that $V_{v_{n}} \in U_{n}$ and thus $\cap V_{v_{n}} \subseteq$ $\bigcap U_{n} . V_{v_{n}}=\left\{f \in F V(X, E):\|f(x)\| \leq 1 / v_{n}(x)\right.$ for all $\left.x \in X\right\}$. Clearly, $\cap\left\{V_{v_{n}}, v_{n} \in\right.$ $V\}$ is absorbent. By Lemma 2.6, $\cap V_{v_{n}}=\left\{f \in F V(X, E):\|f(x)\| \leq \inf _{n}\left(1 / v_{n}(x)\right)\right\}$. If we set $\inf _{n}\left(1 / v_{n}\right)=s(n)$ and assume that the conditions in Lemma 2.7 are all satisfied, then there is a smallest weight $v_{s}(n)$ greater than $1 / s(n)$ and $\cap V_{v_{n}}=V_{v_{s(n)}}$. Furthermore, if $V \sim V^{*}, v_{s(n)} \in V^{*}$ thus $V_{v_{s}}$ and hence $U$ is a neighborhood of zero in $F V(X, E)=F V^{*}(X, E)$ and thus $F V(X, E)$ is countably quasi-barrelled. Conversely, assume that $F V(X, E)$ is countably quasi-barrelled.

We show that $V \sim V^{*}$ under the same conditions in the remark above. First, it is clear that $V \leq V^{*}$ from the construction of $V^{*}$. Also, since $F V(X)$ is assumed to be countably barrelled, then $U=\bigcap U_{n}$ is a $W_{v}$ neighborhood of zero. Hence there is a $v \in V$ such that $V_{v} \subseteq \bigcap\left\{U_{n}, n \in \mathbb{N}\right\}$. But since $V_{v_{s(n)}}=\bigcap V_{v_{n}} \subseteq \bigcap U_{n}$ and $v_{s(n)}$ is the smallest weight greater than $1 / s(n)$ and in view of the fact that $U$ is arbitrary, we can choose $v$ such that $V_{v} \subseteq V_{v_{s(n)}}$ and thus, by Lemma 2.7, $v_{s(n)} \leq v$. Hence, $V^{*} \leq V$. Therefore $V \sim V^{*}$, thus we have the following theorem.

THEOREM 3.4. Let $V$ be a Nachbin family on $X$ such that $\sup _{n} v_{n} \in B_{0}(X)$ for each $\left(v_{n}\right) \in V$ and for every subset $M$ in $F V(X, E), \inf _{f \in M}\|f(x)\|$ is usc on $X$, then $F V(X, E)$ is countably quasi-barrelled if and only if $V \sim V^{*}$.

Similarly, in view of Lemma 2.8, we have the following result.

THEOREM 3.5. Let $V$ be a Nachbin family on $X$ such that $\sup _{n} v_{n} \in B_{0}(X)$ for each $\left(v_{n}\right) \in V$. Assume that $E$ is a normed linear space and either $V \leq B_{0}(X)$ or $X$ is locally compact. If for every subset $M$ in $F V_{0}(X, E), \inf _{f \in M}\|f(x)\|$ is usc on $X$, then $F V_{0}(X, E)$ is countably quasi-barrelled if and only if $V \sim V^{*}$.

Consequently, the following results follow from Theorems 3.4 and 3.5 , respectively.

Proposition 3.6. Let $V$ be a Nachbin family on $X$ such that $\sup _{n} v_{n} \in B_{0}(X)$ for each $\left(v_{n}\right) \in V$, then $C V(X, E)$ is countably quasi-barrelled if and only if $V \sim V^{*}$.

Proposition 3.7. Let $V$ be a Nachbin family on $X$ such that $\sup _{n} v_{n} \in B_{0}(X)$ for each $\left(v_{n}\right) \in V$. Assume that either $V \leq B_{0}(X)$ or $X$ is locally compact, then $C V_{0}(X, E)$ is countably quasi-barrelled if and only if $V \sim V^{*}$. 
The following definition is needed at this point.

Definition 3.8. A subset $B$ of a topological space $X$ is said to be $F(X)$-quasicompact if every function $f$ in $F(X, E)$ bounded on $B$ is in $B_{0}(X, E)$.

Clearly, every $F(X)$-quasicompact set is $F(X)$-pseudocompact and the converse need not be true.

REMARK 3.9. It should be observed from the construction of $V^{*}$ that $V \leq V^{*}$ is always valid. Suppose that

$$
V=\chi_{c}(X)=\left\{\lambda \chi_{K}: \lambda \geq 0 \text { and } K \subset X, K \text { is compact }\right\},
$$

where $\chi_{K}$ is the characteristic function of $K$. It is well known that $C V_{0}(X, E)=(C(X, E)$, c-op). We show when $V^{*} \leq V$. This is true when every $\sup _{v_{n} \in V} v_{n}$ which vanishes at infinity on $X$ is in $V$, that is, $\sup _{v_{n} \in V} v_{n}$ is usc and also has a compact support. Let $v_{n} \in V$ and assume that the compact support of $v_{n}$ is $K_{n}$ for each $n$. Then $\sup _{v_{n} \in V} v_{n}$ is bounded, and by assumption vanishes at infinity, on $\bigcup K_{n}$. Thus, in view of Proposition 3.7, we have the following result.

COROLLARY 3.10. Let $X$ be a completely regular Hausdorff space. Then $(C(X, E)$, c-op) is countably quasi-barrelled if and only if every $F(X)$-quasicompact subset of $X$, which is a countable union of compact subsets of $X$, is compact.

The following example shows that an $F(X)$-pseudocompact set need not be $F(X)$ quasicompact. Let $W$ be the space of ordinals less than the first uncountable ordinals, and let $T$ be the Thychonov plank. Then $T=\left(\bigcup_{n=1}^{\infty}(W \times n)\right)$. $T$ is $F(X)$-pseudocompact but not compact and hence $C(T)$ is not countably barrelled [4, Example 4, page 142]. Also $\chi_{T}$ is bounded on $T$ but is clearly not in $B_{0}(T)$. Hence $T$ is not $F(X)$-quasicompact.

However, we are yet to construct an example of a countably quasi-barrelled space, which is not countably barrelled, using the above result.

REMARK 3.11. If $X$ is a locally compact space and if $E$ is a Banach space and $\chi_{c}(X) \leq V$, it is well known that $C V_{0}(X, E)$ is complete and since a complete countably quasi-barrelled space is countably barrelled, then in view of Proposition 3.7, we have conditions under which $V^{*}$ can also characterize countably barrelled weighted spaces as shown in the next result.

Proposition 3.12. Let $V$ be a Nachbin family on a locally compact space $X$ such that $\sup _{n} v_{n} \in B_{0}(X)$ for each $\left(v_{n}\right) \in V$ where $E$ is a Banach space and $\chi_{c}(X) \leq V$, then the following are equivalent:

(i) $C V_{0}(X, E)$ is countably quasi-barrelled;

(ii) $C V_{0}(X, E)$ is countably barrelled;

(iii) $V \sim V^{*}$.

$(C(X, E), \mathrm{c}-\mathrm{op})$ is countably barrelled if and only if every $F(X)$-pseudocompact subset of $X$, which is a countable union of compact subsets of $X$, is compact [10, Corollary 2.6]. Since $(C(X, E)$, c-op) is complete, and a complete countably quasi-barrelled space is countably barrelled, then as a consequence of Proposition 3.12, we have the following result. 
COROLLARY 3.13. Let $X$ be a locally compact space and $E$ a Banach space. Then the following are equivalent:

(i) $(C(X, E), c-o p)$ is countably quasi-barrelled;

(ii) $(C(X, E), c-o p)$ is countably barrelled;

(iii) every $F(X)$-pseudocompact subset of $X$, which is a countable union of compact subsets of $X$, is compact;

(iv) every $F(X)$-quasicompact subset of $X$, which is a countable union of compact subsets of $X$, is compact;

(v) $V \sim V^{*}$.

Consequently, Corollary 3.13 gives a condition as to when an $F(X)$-pseudocompact set in $X$ is $F(X)$-quasicompact.

COROLlary 3.14. Let $X$ be a locally compact space and $E$ a Banach space. If $(C(X, E), c-o p)$ is countably quasi-barrelled, then every $F(X)$-pseudocompact is $F(X)$ quasi-compact.

REMARK 3.15. We now consider the countably quasi-barrelledness of $C_{b}(X, E)$ equipped with the strict topology $(\beta)$. Since $V \sim B_{0}(X)$ is the defining Nachbin family on $X$ for $\beta$, in view of Proposition 3.7, we show when $V^{*} \leq V$. It is sufficient to show when $\sup _{v_{n} \in V} v_{n}$ is usc. This is the case when the countable union of closed sets in $X$ is closed. Thus we have the following result.

COROLLARY 3.16. Let $X$ be a completely regular Hausdorff space. Then $\left(C_{b}(X, E), \beta\right)$ is countably quasi-barrelled if and only if every countable union of closed sets in $X$ is closed.

If $E$ is complete and $X$ is a locally compact space, it is well known that $\left(C_{b}(X, E), \beta\right)$ is complete. The fact that $\left(C_{b}(X, E), \beta\right)$ is countably barrelled if and only if every countable union of compact sets in $X$ is relatively compact [10, Corollary 2.8] coupled with the fact that a complete countably quasi-barrelled space is countably barrelled, gives the following result as a consequence of Corollary 3.16.

COROLLARY 3.17. Let $X$ be a locally compact space and $E$ a Banach space. Then the following are equivalent:

(i) $\left(C_{b}(X, E), \beta\right)$ is countably quasi-barrelled;

(ii) $\left(C_{b}(X, E), \beta\right)$ is countably barrelled;

(iii) every countable union of closed sets in $X$ is closed;

(iv) every countable union of compact sets in $X$ is relatively compact.

REMARK 3.18. Thus if $X$ is a metrizable locally compact space and $E$ is a Banach space, then $\left(C_{b}(X, E), \beta\right)$ is countably quasi-barrelled and also countably barrelled.

The following example, taken from [2], which we use to show that a countably quasi-barrelled space need not be countably barrelled, was communicated to us by Ian Tweddle. The ordinary $l^{\infty}(S)$ ( $S=$ positive integers) is just the bounded (continuous) functions on the discrete space $\mathbb{N}$ of natural numbers. According to Collins, its dual under the strict topology is $l^{1}$. The closed unit ball of $l^{1}$ is separable and bounded for the pairing of $l^{\infty}$ and $l^{1}$, and if $l^{\infty}$ is countably barrelled under the strict topology, the closed unit ball of $l^{1}$ is equicontinuous and so, being closed, it is weakly compact 
for the weak topology defined by $l^{\infty}$, which is false, since $l^{1}$ is not reflexive under the weak topology. So $l^{\infty}$ is not countably barrelled but it is countably quasi-barrelled by Corollary 3.16.

4. Quasi-barrelledness of weighted spaces. In view of the fact that a separable or metrizable countably quasi-barrelled space is quasi-barrelled, the quasi-barrelledness of $C V(X, E)$ and $C V_{0}(X, E)$ follow from Propositions 3.6 and 3.7, respectively. Thus we have the following results.

Proposition 4.1. Let $V$ be a Nachbin family on $X$ such that $\sup _{n} v_{n} \in B_{0}(X)$ for each $\left(v_{n}\right) \in V$. If $C V(X, E)$ is metrizable or separable, then $C V(X, E)$ is quasi-barrelled if and only if $V \sim V^{*}$.

Proposition 4.2. Let $V$ be a Nachbin family on $X$ such that $\sup _{n} v_{n} \in B_{0}(X)$ for each $\left(v_{n}\right) \in V$. Assume that either $V \leq B_{0}(X)$ or $X$ is locally compact. If $C V_{0}(X, E)$ is separable or metrizable, then $C V_{0}(X, E)$ is quasi-barrelled if and only if $V \sim V^{*}$.

Conditions under which weighted spaces are metrizable or separable are well known. For example, see [8, 9].

Specifically, we have the following result.

COROLLARY 4.3. Let $X$ be separably submetrizable, then the following are equivalent:

(i) $\left(C_{b}(X, E), \beta\right)$ is countably quasi-barrelled;

(ii) every countable union of closed sets in $X$ is closed;

(iii) $\left(C_{b}(X, E), \beta\right)$ is quasi-barrelled.

Proof. The proof is easy in view of Corollary 3.16 and the fact that if $X$ is separably submetrizable, then $\left(C_{b}(X, E), \beta\right)$ is separable, see [9].

For the definition of separably submetrizable, see [9] or [10].

REMARK 4.4. There are other conditions for which a weighted countably quasibarrelled space is quasi-barrelled. For example if the separability or metrizability condition on $C V(X, E)$ and $C V_{0}(X, E)$ in Propositions 4.1 and 4.2 is replaced with the condition that the weighted spaces have a bounded absorbing sequence of metrizable subsets (see [4, Corollary 9, page 137]). Of particular importance of this result is its application to the study of countably quasi-barrelled inductive limits of weighted spaces, which leads to results of special interest.

Let $\mathscr{V}=\left(v_{n}, n=1,2, \ldots\right)$ be a decreasing sequence of strictly positive continuous weights on $X . V_{n}$ is a system of weights $\left(a v_{n}, a>0\right)$ for each $n$ and we write $C\left(v_{n}\right)(X, E)$ and $C\left(v_{n}\right)_{0}(X, E)$ instead of $C\left(V_{n}\right)(X, E)$ and $C\left(V_{n}\right)_{0}(X, E)$, respectively. Denote the weighted inductive limit as

$$
\begin{gathered}
\mathscr{V} C(X, E)=\operatorname{ind}\left(C\left(v_{n}\right)(X, E), n=1,2, \ldots\right), \\
\mathscr{V}_{0} C(X, E)=\operatorname{ind}\left(C\left(v_{n}\right)_{0}(X, E), n=1,2, \ldots\right) .
\end{gathered}
$$

Define the following system of weights associated to $\mathscr{V}$ as $\bar{V}=\{\bar{v}: X \rightarrow \mathbb{R}: \bar{v}$ is a weight on $X$ such that $\bar{v} / v_{n}$ is bounded from above on $X$ for each $n$ \}. 
The weights of the form $v(t)=\inf \left(a(n) v_{n}(t): n=1,2, \ldots\right)$ constitute a directed fundamental family of members of $\bar{V}$. If $X$ is locally compact then $\mathscr{V}_{0} C(X, E)$ is a dense topological subspace of $C \bar{V}_{0}(X, E)$, and if $E$ is complete then $C \bar{V}_{0}(X, E)$ is the completion of $\mathscr{V}_{0} C(X, E)$. Similarly $C \bar{V}(X, E)=\mathscr{V} C(X, E)$ algebraically. Those are well known, for example see [11].

We show the conditions under which $\bar{V}^{*} \sim \bar{V}$. It is sufficient to show when $\bar{V}^{*} \leq \bar{V}$. Let $\bar{v}_{p}=\inf _{n \in \mathbb{N}} \alpha_{n}^{p} v_{n} \in \bar{V}$ and assume $\sup _{p \in \mathbb{N}} \bar{v}_{p} \in B_{0}(X)$

$$
\sup _{p \in \mathbb{N}} \bar{v}_{p}=\sup _{p \in \mathbb{N}} \inf _{n \in \mathbb{N}} \alpha_{n}^{p} v_{n}=\inf _{n \in \mathbb{N}} \sup _{p \in \mathbb{N}} \alpha_{n}^{p} v_{n}
$$

Set $\sup _{p \in \mathbb{N}} \alpha_{n}^{p}=\lambda_{n}$. Since $\sup _{p \in \mathbb{N}} \bar{v}_{p} \in B_{0}(X), \lambda_{n}$ is finite and hence

$$
\sup _{p \in \mathbb{N}} \bar{v}_{p}=\inf _{n \in \mathbb{N}} \lambda_{n} v_{n} \in \bar{V} .
$$

If for every $\bar{v} \in \bar{V}$ there is a continuous weight $\tilde{v} \in \bar{V}$ such that $\bar{v} \leq \tilde{v}$, then it is clear that $\bar{V}^{*} \leq \bar{V}$. Since $C \bar{V}(X, E)$ and $C \bar{V}_{0}(X, E)$ each have a bounded absorbing sequence of metrizable subsets $C\left(v_{n}\right)(X, E)$ and $C\left(v_{n}\right)_{0}(X, E)$, respectively. In view of Remark 4.4, Propositions 4.1 and 4.2, respectively, give the following.

Proposition 4.5. Let $X$ be a completely regular Hausdorff space and $\mathscr{V}=\left(v_{n}\right)$ a sequence of continuous weights on $X$ such that $\left(v_{n}\right) \in B_{0}(X)$. If for every $\bar{v} \in \bar{V}$, there is a continuous weight $\tilde{v} \in \bar{V}$ such that $\bar{v} \leq \tilde{v}$, then $C \bar{V}(X, E)$ is quasi-barrelled.

Proposition 4.6. Let $X$ be a locally compact space and $\mathscr{V}=\left(v_{n}\right)$ a sequence of continuous weights on $X$ such that $\left(v_{n}\right) \in B_{0}(X)$. If for every $\bar{v} \in \bar{V}$, there is a continuous weight $\tilde{v} \in \bar{V}$ such that $\bar{v} \leq \tilde{v}$, then $C \bar{V}_{0}(X, E)$ is quasi-barrelled.

5. (DF)-weighted spaces. A countably quasi-barrelled space with a fundamental sequence of bounded sets is a (DF)-space. Thus all our results on countably quasibarrelled weighted spaces and quasi-barrelled weighted spaces are also true for (DF)weighted spaces provided that those spaces have a fundamental sequence of bounded sets. Thus for example, according to Corollary 3.16, if $X$ is a completely regular Hausdorff space, then $\left(C_{b}(X, E), \beta\right)$ is a (DF)-space if and only if every countable union of closed sets in $X$ is closed. If $X=R,\left(C_{b}(X, E), \beta\right)$ is then not a (DF)-space and thus a $(g \mathrm{DF})-\left(C_{b}(X, E), \beta\right)$ need not be a (DF)-space [5, pages 266, 269]. Of special interest is Proposition 4.5. Since $C \bar{V}(X, E)$ has a fundamental sequence of bounded sets, then, under the assumptions in the proposition, $C \bar{V}(X, E)$ is also a (DF)-space. This, in the light of application, is exactly [1, Theorem 1.8] and of course the main result in that paper with a more technical proof. Thus the results, which are true for quasi-barrelled $C \bar{V}(X, E)$, are also true for countably quasi-barrelled or (DF)- $C \bar{V}(X, E)$.

6. Countable neighborhood property (cnp). A space $E$ is said to satisfy the cnp if, given any sequence $\left(U_{n}, n=1,2, \ldots\right)$ of zero neighborhoods in $E$, there is $a(n)>0$ such that $U=\bigcap a(n) U_{n}: n=1,2, \ldots$ is a neighborhood of zero (see [11, Definition 8.3.4]). In view of Propositions 3.6 and 3.7, a close observation of the construction of $V^{*}$ in Remark 3.3 gives, respectively, the following results which are generalizations of [11, Proposition 8.3.5]. 
Proposition 6.1. Let $V$ be a Nachbin family on a completely regular Hausdorff space $X$ such that $\sup _{n} v_{n} \in B_{0}(X)$ for each $\left(v_{n}\right) \in V$, then a countably quasi-barrelled $C V(X, E)$ satisfies cnp.

Proposition 6.2. Let $V$ be a Nachbin family on $X$ such that $\sup _{n} v_{n} \in B_{0}(X)$ for each $\left(v_{n}\right) \in V$. If either $V \leq B_{0}(X)$ or $X$ is locally compact, then a countably quasibarrelled $C V_{0}(X, E)$ satisfies cnp.

ACKNOWLEDGments. I am grateful to Abdul Salam ICTP for hospitality and Ian Tweddle whose careful reading, suggestions, and corrections of our previous work led to the writing of this paper. I am also grateful to the referee for his helpful comments and suggestions.

\section{REFERENCES}

[1] J. Bonet, A projective description of weighted inductive limits of spaces of vector valued continuous functions, Collect. Math. 34 (1983), no. 2, 115-125.

[2] H. S. Collins, On the space $l^{\infty}(S)$, with the strict topology, Math. Z. 106 (1968), 361-373.

[3] B. Ernst and P. Schnettler, On weighted spaces with a fundamental sequence of bounded sets, Arch. Math. (Basel) 47 (1986), 552-559.

[4] T. Husain and S. M. Khaleelulla, Barrelledness in Topological and Ordered Vector Spaces, Lecture Notes in Mathematics, vol. 692, Springer-Verlag, Berlin, 1978.

[5] H. Jachow, Locally Convex Spaces, B. G. Teubner, Stuttgart, 1981.

[6] G. Köthe, Topological Vector Spaces. I, Springer-Verlag, New York, 1969.

[7] L. Nachbin, Topological vector spaces of continuous functions, Proc. Nat. Acad. Sci. U.S.A. 40 (1954), 471-474.

[8] J. O. Olaleru, Completeness and metrizability in weighted spaces of continuous functions, J. Sci. Res. Dev. 3 (1998), 177-183.

[9] _ Separability in weighted spaces of continuous functions, J. Sci. Res. 3 (1998), 89-94.

[10]_, Barrelled weighted locally convex spaces, preprint, 2001.

[11] P. Pérez Carreras and J. Bonet, Barrelled Locally Convex Spaces, North-Holland Mathematics Studies, vol. 131, North-Holland Publishing, Amsterdam, 1987.

[12] J. B. Prolla, Weighted spaces of vector-valued continuous functions, Ann. Mat. Pura Appl. (4) 89 (1971), 145-157.

[13] J. Schmets, Espaces de Fonctions Continues, Lecture Notes in Mathematics, vol. 519, Springer-Verlag, Berlin, 1976.

[14] W. H. Summers, A representation theorem for biequicontinuous completed tensor products of weighted spaces, Trans. Amer. Math. Soc. 146 (1969), 121-131.

J. O. Olaleru: Mathematics Department, University of LAgos, YABA, LAgos, Nigeria E-mail address: ola1eruj@netscape.net 


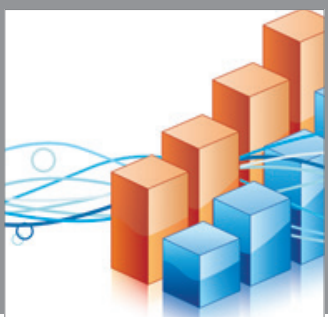

Advances in

Operations Research

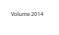

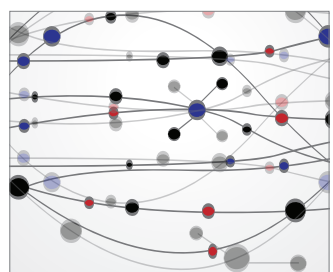

\section{The Scientific} World Journal
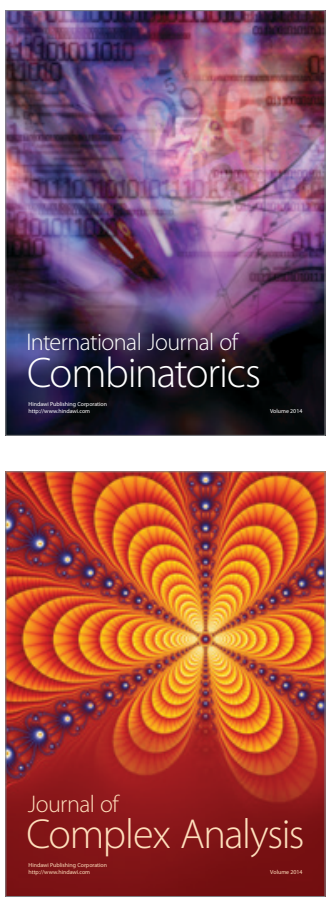

International Journal of

Mathematics and

Mathematical

Sciences
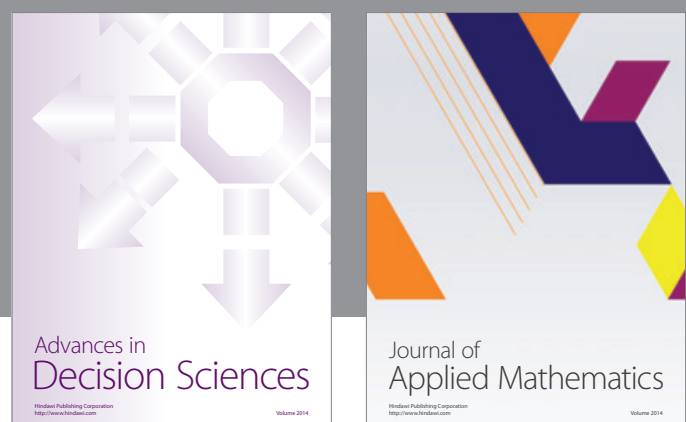

Journal of

Applied Mathematics
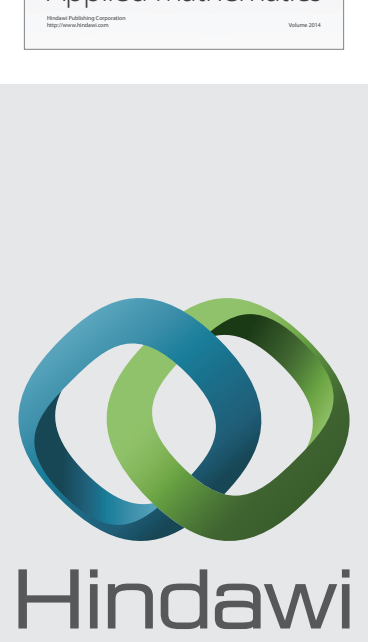

Submit your manuscripts at http://www.hindawi.com
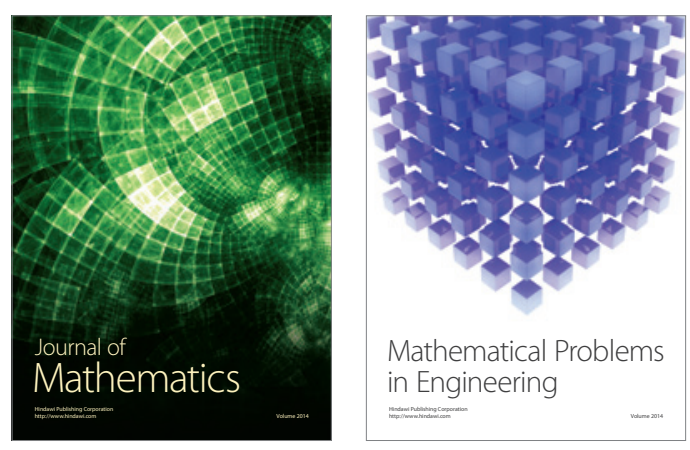

Mathematical Problems in Engineering
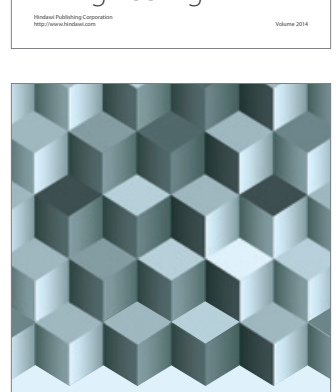

Journal of

Function Spaces
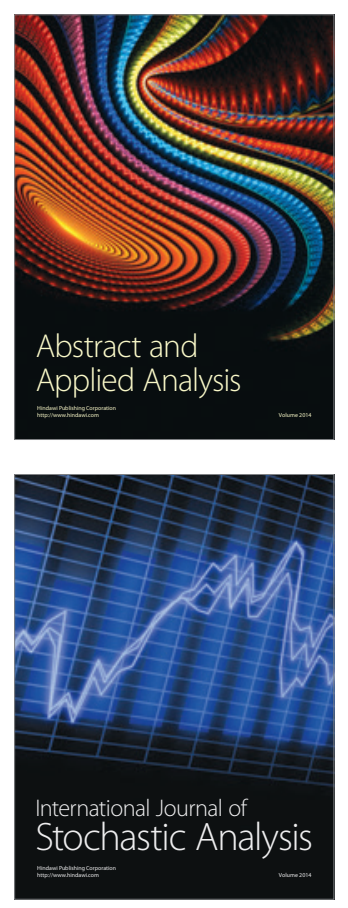

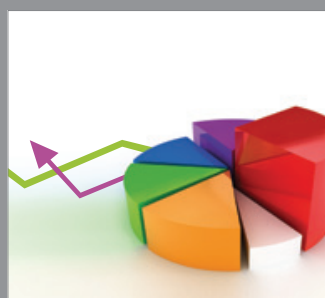

ournal of

Probability and Statistics

Promensencen
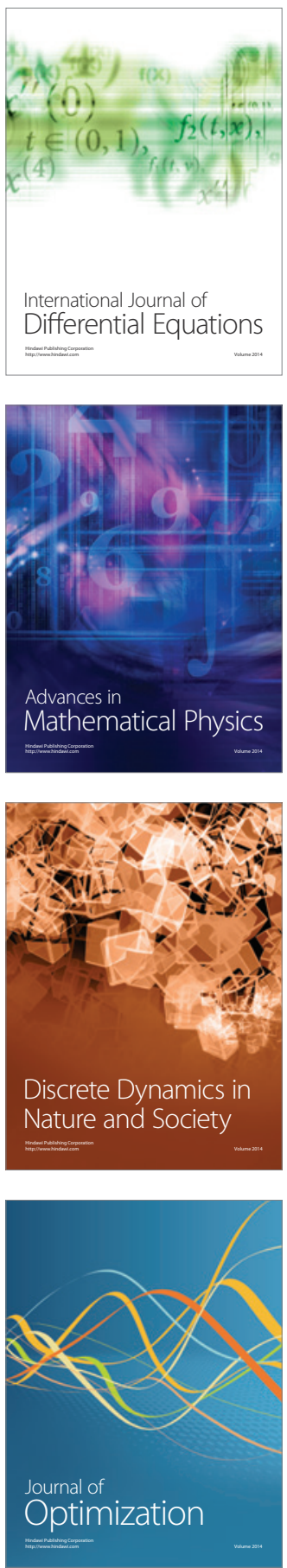